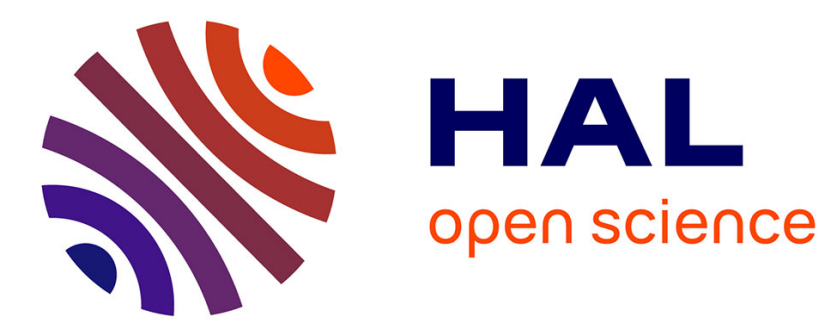

\title{
Influence des hétérogénéités de déformation élastique sur l'élargissement des pics de diffraction $\mathrm{X}$
}

\author{
P. Mabelly, P. Hadmar, M. Desvignes, J. Sprauel
}

\section{To cite this version:}

P. Mabelly, P. Hadmar, M. Desvignes, J. Sprauel. Influence des hétérogénéités de déformation élastique sur l'élargissement des pics de diffraction X. Journal de Physique IV Proceedings, 1996, 06 (C4), pp.C4-197-C4-209. 10.1051/jp4:1996418 . jpa-00254301

\section{HAL Id: jpa-00254301 https://hal.science/jpa-00254301}

Submitted on 1 Jan 1996

HAL is a multi-disciplinary open access archive for the deposit and dissemination of scientific research documents, whether they are published or not. The documents may come from teaching and research institutions in France or abroad, or from public or private research centers.
L'archive ouverte pluridisciplinaire HAL, est destinée au dépôt et à la diffusion de documents scientifiques de niveau recherche, publiés ou non, émanant des établissements d'enseignement et de recherche français ou étrangers, des laboratoires publics ou privés. 


\title{
Influence des hétérogénéités de déformation élastique sur l'élargissement des pics de diffraction $X$
}

\author{
P. Mabelly, P. Hadmar, M. Desvignes et J.M. Sprauel
}

ENSAM, Laboratoire MECASURF, 2 cours des Arts et Métiers, 13617 Aix-en-Provence cedex 1, France

\begin{abstract}
Résumé: Dans les études portant sur l'élargissement des pics de diffraction des rayons $\mathrm{X}$ des matériaux polycristallins, l'influence des hétérogénéités de déformation élastique ou "des contraintes internes" a souvent été négligée au profit des effets bien connus de taille et de distorsion introduits par Warren et Averbach [1,2]. Cette étude a pour but de quantifier tant par le calcul que par l'expérimentation l'influence de ces hétérogénéités de déformation sur l'élargissement. L'utilisation de calculs micromécaniques dérivés des modèles de Kröner [3] et do Hill [4] a permis de caractériser l'influence des hétérogénéités de déformation élastique sur l'élargissement dans les domaines élastique et plastique. Au vu de cette étude, l'importance de cette source d'élargissement ne peut plus dès lors être négligée et semble être, au moins pour la sollicitation et l'acier inoxydable austénitique 316Ti étudiés, une importante source d'élargissement.
\end{abstract}

\begin{abstract}
In the study of the diffraction peak broadening of massive polycrystalline metals, the effect of strain heterogeneity among coherently diffracting domains has been often neglected in favour of the well known size and distortion effects introduced by Warren and Averbach [1,2]. This study is aimed at weighting the effect of the strain heterogeneity by numerical calculation and experimentation. The use of micromechanical calculation introduced first by Kröner [3] and Hill [4] has led to the characterisation of the strain heterogeneity inside diffracting volume. The strain effect has proved to play a major role in the diffraction peak broadening of the austenic stainless steel used for the experimental study (316Ti).
\end{abstract}

\section{INTRODUCTION}

$\mathrm{La}$ variation de la largeur des pics de diffraction des rayons $\mathrm{X}$ des matériaux métalliques massifs, au cours de sollicitations mécaniques, est un phénomène connu depuis maintenant une cinquantaine d'années. De nombreuses études expérimentales se sont attachées à observer les variations des largeurs des pics de diffraction pour divers types d'essais mécaniques simples (traction, torsion, fatigue oligocyclique en traction-compression...), mais aucune analyse véritable des sources de cet élargissement n'a été entreprise pour expliquer de manière spécifique cet élargissement dans le cas des matériaux métalliques polycristallins.

La largeur et la forme des pics de diffraction des matériaux polycristallins peuvent être dues à quatre phénomènes complémentaires [5] :

- L'élargissement instrumental dû à la géométrie du faisceau (divergence...), aux conditions de diffraction (décalages angulaires, défauts géométriques... ), et à la fonction spectrale de la source de rayons $\mathrm{X}$.

- La taille des domaines cohérents de diffraction et leurs déformations élastiques internes [1].

- La distribution des compositions des domaines cohérents (ségrégations...) qui influe sur la distribution des distances interréticulaires.

- L'Hétérogénéité des Déformations Elastiques (HDE) des domaines cohérents de diffraction. Cette hétérogénéité a pour origine les caractéristiques anisotropes du monocristal et les incompatibilités plastiques entre domaines cohérents $[5,6]$. 
L'importance relative des différents effets, hormis celle de l'élargissement instrumental est méconnue. Nombreuses sont les études qui se sont limitées à appliquer les résultats de diffractométrie des poudres à cette étude de "matériaux polycristallins massifs". L'analyse de Warren et Averbach, qui comme nous allons le rappeler dans notre chapitre 2 est bien spécifique à cette analyse des poudres, s'attache à quantifier le deuxième effet d'élargissement du à la distorsion des déformations élastiques et à la taille des cristallites. Cette méthode est devenue malgré tout avec le temps, un élément incontournable de l'analyse dite "fine" de ces élargissements de pics de diffraction de matériaux pourtant massifs. Dans cet exposé, nous introduirons tout d'abord les différents effets connus d'élargissement et mettrons en évidence l'importance de la connaissance des hétérogénéités de déformation. Notre étude consiste en la généralisation au domaine plastique des matériaux de type CFC de l'étude effectuée par Sprauel et Castex [5, 6, 7] de l'élargissement sous chargement élastique des pics de diffraction. Aussi, nous ne présenterons que les hypothèses ayant servi à la construction des calculs élastoplastique et une comparaison de leurs résultats avec l'expérimentation.

\section{L'ELARGISSEMENT DES PICS DE DIFFRACTION $\mathbf{X}$}

La diffraction des rayons $\mathrm{X}$ provient de la diffusion cohérente du faisceau auquel est exposé une structure périodique. Comme tout phénomène d'interférence, la diffraction des rayons $\mathrm{X}$ n'est possible que lorsque la périodicité des éléments diffusants, (atomes molécules ou ions) est intacte ou faiblement altérée. Les discontinuités du réseau tels que les joints de grains, les dislocations, les lacunes, auront donc une grande influence : ils ne participeront pas à la diffraction mais délimiteront les domaines sans défauts qui y participeront $[5,8]$. Nous appellerons désormais ces domaines de la structure du matériau Domaines Cohérents de Diffraction (DCD). La diffraction des rayons $X$ par les matériaux métalliques usuels, étant caractérisée par le rôle prépondérant des diffractions de premier ordre, c'est à dire, interne aux DCD et non entre DCD, nous pourrons considérer la formation du pic de diffraction comme l'addition ou la superposition des fonctions intensité élémentaire créés par chacun des DCD du matériau.

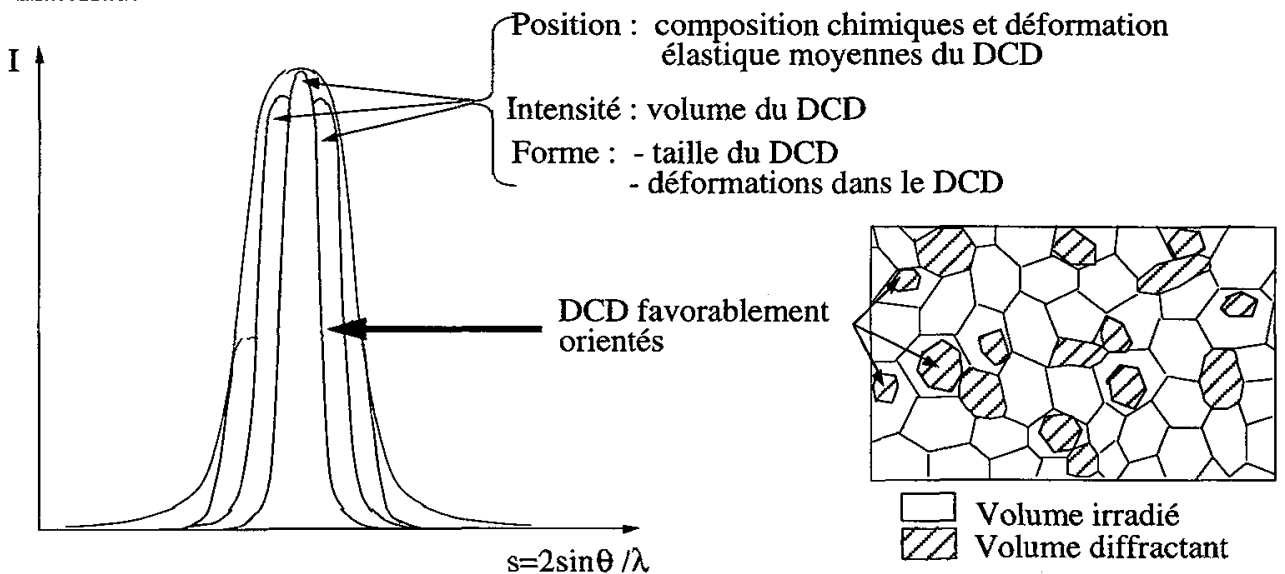

Figure 1: Composition des différents effets d'élargissement Superposition of the broadening effect

Chaque domaine cohérent sera défini par la donnée de sa position $P$, et de son orientation $\Omega$ par rapport à un repère fixe, de sa forme et de son état mécanique local (champ de déformation local, caractéristiques élastiques,...). Un DCD sera en position de diffraction constructive lorsque la normale aux plans réticulaires d'indices de Miller $h, k, l$ sera colinéaire au vecteur diffraction et que la distance interréticulaire $d_{h k l}$ respectera la loi de Bragg [5]: 


$$
\lambda=2 d_{h k l}(P, \Omega) \sin \left(\theta_{h k l}(P, \Omega)\right)
$$

où $\lambda$ est la longueur d'onde du faisceau monochromatique incident et $\theta_{h k l}$ l'angle de diffraction.

La distance interréticulaire utilisée dans la relation (1) correspond en fait à la moyenne arithmétique des distances interréticulaires de l'empilement de plan d'indice $h, k, l$ participant à la diffraction. Les conditions de diffraction ne permettent pas de définir de manière unique l'orientation des cristallites en position de diffraction : une rotation $\beta$ du repère cristallographique autour de la normale $\mathbf{n}$ au plan diffractant ou direction de mesure conserve en effet les conditions de Laue.

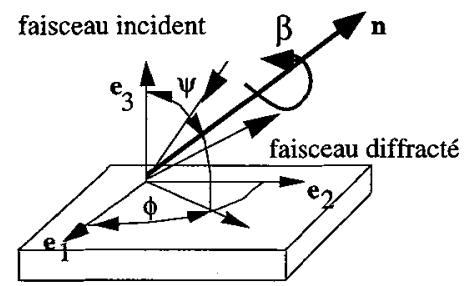

Figure 2 : Définition du repère de la pièce et des angles $\phi$, $\psi$ et $\beta$

Position of the different angles in the piece's coordinate system

Dans les matériaux métalliques polycristallins, où les DCD abondent, de très nombreux cristallites, définis à une rotation près autour de la direction de mesure seront simultanément en position de diffraction. Cet ensemble de cristallites constitue un volume discontinu de matière que nous appellerons désormais volume diffractant.

Si l'on considère maintenant un état mécanique initial non déformé et non contraint du matériau, nous pouvons définir une distance $d_{0 h k l}$ caractéristique du seul arrangement des éléments diffusant et des variations de composition chimique locale (ségrégation, ...). Avec $d_{0 h k l}$ ainsi défini, nous pourrons écrire la formule [5] :

$$
d_{h k l}(P, \Omega)=d_{0 h k l}(P, \Omega)\left[1+\varepsilon_{h k l}(P, \Omega)\right]
$$

où $\varepsilon_{h k l}$ est la valeur moyenne sur l'empilement de plan d'indice $h, k, l$ composant le DCD, de la déformation suivant la normale au plan $\{\mathrm{hkl}\}$.

Cette déformation est le résultat de la superposition des déformations élastique et thermique du réseau. Il est remarquable de noter que la déformation plastique ne peut intervenir dans la composition de $\varepsilon_{h k l}$. En effet, la déformation plastique provient du mouvement et de la multiplication de défauts cristallins externes aux DCD (lacunes, dislocations, ...) qui laissent le réseau inchangé après leur passage dans le DCD. Le pic de diffraction X provenant de l'image dans l'espace réciproque du réseau cristallin, la diffraction des rayons $\mathrm{X}$ ne peut donc accéder à la partie plastique des déformations affectant le DCD. La déformation plastique est par contre d'une importance capitale pour deux raisons :

- Les défauts nés de la déformation plastique constitueront les limites des DCD. De la distance moyenne entre défauts, dépendra la taille moyenne des DCD.

- D'autre part, la déformation plastique étant une déformation libre de contrainte, elle est source d'incompatibilités qui entraîneront la formation d'un champ de déformation élastique non compatible. Ce champ de déformation élastique est le dual d'un champ de "contraintes internes" [6].

A chacun des DCD, nous pouvons appliquer la théorie de Warren et Averbach $[1,2]$ et redéfinir les effets de taille et de distorsion. L'application de cette théorie à l'ensemble des cristallites est par contre sans fondement : l'état de déformation moyen de chaque cristallite n'est absolument pas homogène dans l'ensemble du volume diffractant. En effet, contrairement aux poudres, les cristallites des matériaux polycristallins massifs sont caractérisés par l'existence de 
contraintes internes non nulles et absolument pas homogènes [9]. L'effet de taille est naturellement lié à une notion de densité de défauts, tandis que l'effet de distorsion est lié à l'homogénéité du champs de déformation interne au cristallite. La fonction intensité diffractée I(s) est obtenue par la somme sur l'ensemble des domaines cohérents des fonctions intensités élémentaires :

$$
I(s)=\sum_{\text {Volume diffractant }} I_{D C D}(s, P, \Omega) \text { où } s=1 / d=2 \sin \theta_{h k l} / \lambda
$$

Cette fonction intensité peut être réécrite en faisant intervenir une fonction de distribution des déformations moyennes dans le volume diffractant $F_{h k l}$ :

$$
I(s)=\sum_{\text {Volume diffactant }} I_{D C D}(s, \varepsilon, P, \Omega)=\int_{\varepsilon_{\min }}^{\varepsilon_{\max }} F_{h k l}(\varepsilon) . I_{D C D}(s, \varepsilon, P, \Omega) d \varepsilon
$$

Si l'on suppose que la fonction intensité élémentaire des DCD est globalement indépendante de $\mathcal{E}$, alors nous pouvons arriver à l'écriture proposée par Sprauel [6]:

$$
I(s)=I_{\varepsilon}(s) * I_{D C D}(s) \text { avec } I_{\varepsilon}(s)=F_{h k l}(\varepsilon) \text { pour } \varepsilon=\frac{\left(s-1 / d_{0 k k l}\right)}{s}
$$

Cette équation montre clairement le rôle joué par les HDE. Plus la distribution des déformations sera large, plus la composition des faisceaux diffractés entrainera un élargissement important. Dans le cas contraire (matériaux recristallisés, détensionnés), les hypothèses initiales de Warren, en l'absence de HDE, peuvent s'appliquer. Pour évaluer la fonction de distribution des déformations $F_{h k l}$, il nous faut maintenant résoudre un des plus vieux problèmes de mécanique hétérogène [10]. Nous allons dans un premier temps définir plus en détail les hypothèses et les conditions de ce calcul micromécanique, puis dans le chapitre 4.3, préciser les conditions de calcul des fonctions de distribution et des fonctions intensité des pics résultants.

\section{MODELISATION ELASTOPLASTIQUE DU MATERIAU}

La résolution d'un "problème d'hétérogénéité" par la théorie de la Mécanique des Milieux Continus (MMC) impose d'emblée l'échelle d'arrivée de la modélisation. Le volume étudié, appelé aussi élément d'arrivée est un élément de volume de la pièce suffisamment petit, pour que les champs de contraintes et de déformations y soient continus et satisfassent aux équations de la MMC et assez grand pour que la loi de comportement obtenue ne fluctue pas "trop" d'un élément de volume à l'autre. De telles conditions sont en général satisfaites ${ }^{1}$, car les hétérogénéités traitées (cristallites, grains, renforts composites) sont de taille largement inférieure à celle de la pièce étudiée (ou de ses sous ensembles). Dans notre application à l'étude de l'influence des hétérogénéités de déformation sur la diffraction des rayons $X$ [11], cette condition est presque redondante avec les clauses d'application de cette technique. Le volume étudié, limité par la dimension d'un faisceau de quelques millimètres carrés et sa pénétration dans le matériau (quelques micromètres), doit en effet comporter un grand nombre de cristallites en position de diffraction et, de plus, être sujet à des gradients de contraintes limités.

L'échelle de départ de notre modélisation dépend par contre de notre capacité à écrire les lois de comportement locales. Une échelle séduisante pour notre application des calculs micromécaniques à l'étude de la diffraction des rayons $\mathrm{X}$ est bien sûr l'échelle de la dislocation. Une telle description permettrait, une fois les champs de déplacement à l'intérieur de chacun des cristallites connus, de calculer facilement la composition des faisceaux diffusés par les atomes.

\footnotetext{
1 Un contre exemple peut être l'application à l'étude du comportement d'une tôle à très gros grains. Une autre formulation de cette règle peut être: "Le matériau doit être suffisament microhétérogène pour être macrohomogène" (règle inspirée par Zaoui [10])
} 
Si le comportement mécanique d'une dislocation est maintenant bien décrit, son mouvement et ses interactions avec les autres dislocations, les systèmes de dislocations (bandes de glissement ou les cellules de dislocation) et même les joints de grains ne sont pas clairement ou même pas du tout décrits. Une autre échelle possible peut être constituée par les zones d'hétérogénéités internes aux grains, les bandes de glissement, les parois ou les cellules de dislocations... Nous nous limiterons cependant à une approche moins microscopique, c'est à dire à l'échelle du cristallite. Son comportement mécanique est en effet relativement bien approché, après quelques adaptations, par celui du monocristal.

Le comportement élastoplastique étant par nature non linéaire, il est étudié classiquement de manière incrémentale [4]. Un incrément de contrainte entraîne un incrément de déformation, ou inversement, et ce de façon "généralement" unique. Dans le cas d'un comportement non visqueux, cette formulation revient à lier, en faisant abstraction du temps, une vitesse de contrainte à une vitesse de déformation. Afin de décrire les conditions d'équilibre, de compatibilité et de continuité nous utiliserons les taux de contraintes nominales notés $\underline{\underline{N}}$ et les composantes du vecteur vitesse en tout point du solide. L'utilisation du tenseur des contraintes nominales permet, au prix d'une complication toute relative des équations de comportement, d'exprimer dans la configuration actuelle (assimilée à la configuration de référence), les conditions aux limites des équations d'équilibre et les relations de transition d'échelles. Afin de distinguer les grandeurs locales et globales, nous utiliserons les notations suivantes:

$\dot{\underline{N}}$ : désignera le tenseur des taux de contraintes nominales macroscopiques,

市: $\quad$ désignera le tenseur des taux de contraintes locales,

$\underline{\underline{G}}=V_{i, j}$ : désignera le gradient du vecteur vitesse macroscopique,

$\underline{\underline{g}}=v_{i, j}:$ désignera le gradient du vecteur vitesse local.

Le comportement mécanique du polycristal est conditionné par le comportement de chacun des cristallites qui le composent et de leurs interactions. Après avoir décrit les hypothèses qui nous ont permis d'écrire leur loi de comportement élastoplastique, nous expliciterons les méthodes de localisation qui permettent d'exprimer les variables locales de chaque cristallite à partir des variables macroscopiques (conditions de chargement, ...). Nous ne parlerons guère des conditions d'homogénéisation car elles reviennent pour les grandeurs choisies à de simples moyennes volumiques des grandeurs locales[12].

\subsection{Comportement élastoplastique d'un cristallite}

Le but de ce paragraphe n'est pas de donner en détail toutes les équations ayant servi de base au calcul élastoplastique. Nous nous contenterons de donner les hypothèses classiques qui permettent d'écrire la loi de comportement suivante $[12,13]$ :

$$
\dot{n}_{i j}(r)=l_{i j k l}(r) . v_{k, l}(r) \text { pour tout point quelconque } P \text { de coordonnées } r
$$

Dans le domaine élastique, nous utiliserons les lois de comportement élastique des monocristaux. Les tenseurs des rigidités élastiques sont en général caractérisés par une grande anisotropie. La figure 3 illustre l'importante évolution du module de Young d'un monocristal suivant la direction de l'espace. On remarquera que le monocristal d'aluminium est caractérisé par la quasi-isotropie de ses caractéristiques élastique (sans égaler toutefois le tungstène).

Afin de diminuer et de simplifier le nombre des mécanismes de déformation dans le domaine plastique, nous limiterons notre étude aux cas des matériaux C.F.C. Le seul mécanisme de déformation des cristallites considéré sera le glissement. Ce principal mode de déformation à froid des métaux s'effectue de façon privilégiée dans certaines directions de plan du monocristal. Dans le cas de la structure C.F.C., les glissements s'effectuent principalement dans les plans denses $\{111\}$ (4 plans) suivant les directions $<110>$ ( 3 directions) de la maille élémentaire. Le glissement pourra intervenir selon un de ces 12 systèmes si la cission critique est atteinte (critère de Schmid). Pour tenir compte de l'écrouissage des cristallites, nous utiliserons les lois d'écrouissage des monocristaux introduites par Franciosi [14]. 

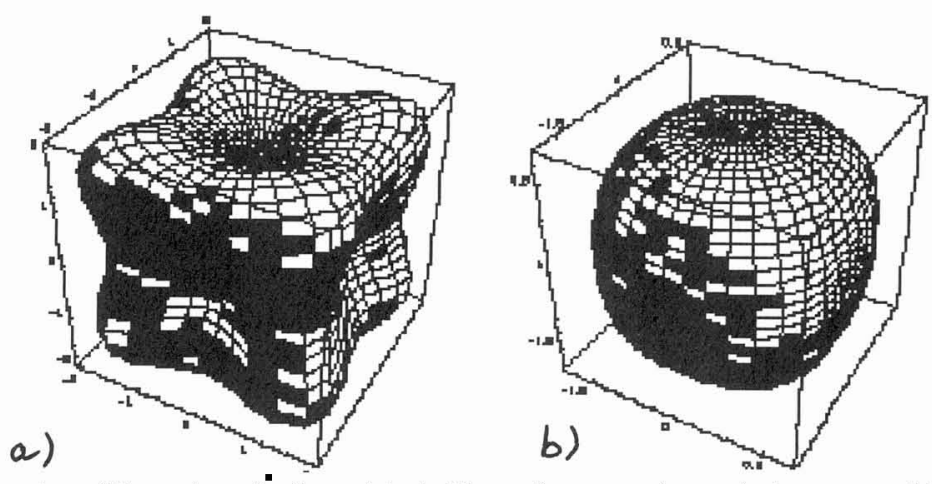

Figure 3: Représentation tridimensionnelle du module de Young des monocristaux de fer $\alpha$ (a) et d'aluminium (b) Spatial representation of the Young modulus of the ferrite and aluminum single crystals

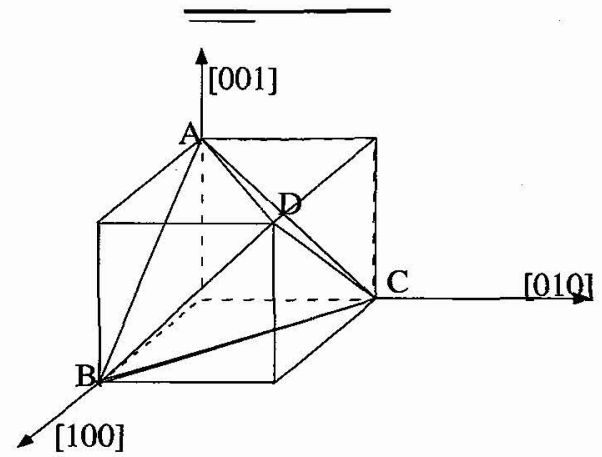

Figure 4: Représentation des systèmes de glissement d'un cristal CFC

The CFC glide system

La figure 4 montre la représentation des 4 plans de glissement et les traces des directions de glissement dans la maille élémentaire. La plasticité induit de par le nombre limité des systèmes de glissement une anisotropie très élevée. Elle est donc appelée à jouer un rôle majeur dans la création des hétérogénéités de déformation. On admettra que l'ensemble des hypothèses détaillées ici nous permet d'écrire la loi de comportement (6) qui lie les tenseurs, taux de contraintes nominales et gradient de vitesse locaux.

\section{2 "Du micro au macro"}

En tout point $P$ de coordonnées $r$ du solide macro-homogène étudié nous devons satisfaire l'équation d'équilibre (7):

$$
\forall P \in V \quad n_{i j, i}=0
$$

L'équation peut être transformée de façon à faire apparaître le tenseur de Green $\Gamma$ du milieu infini macro-homogène équivalent. A cette fin, nous décomposerons le tenseur $\underline{\underline{a}}$ en deux termes : l'un uniforme $\underline{\underline{L}}$ et l'autre variant localement noté $\underline{\delta l_{(r)}}$. 


$$
\begin{gathered}
\left(\delta l_{i j k l(r)} v_{k, l(r)}\right)_{, i}+L_{i j k l}^{0} v_{k, l i(r)}=0 \\
\Downarrow \\
\left\{\Gamma_{j m\left(r-r^{\prime}\right)}^{*} L_{i j k l}^{0} v_{k, l(r)}-v_{j(r)} L_{l k j i}^{0} \Gamma_{k m, l\left(r-r^{\prime}\right)}^{*}\right\}_{, i}+\Gamma_{j m\left(r-r^{\prime}\right)}^{*}\left(\delta l_{i j k l(r)} v_{k, l}\right)_{, i}-v_{m(r)} \delta\left(r-r^{\prime}\right)=0
\end{gathered}
$$

Cette équation conduit, après intégration sur le volume et dérivation du champ de vitesse obtenu à l'expression suivante du gradient des vitesses:

$$
g_{m n}(r)=g_{m}^{0}(r)+\int_{V} \Gamma_{j m, i n\left(r^{\prime}-r\right)}^{*} \delta l_{i j k l\left(r^{\prime}\right)} g_{k l\left(r^{\prime}\right)} d V
$$

\subsection{Approximation autocohérente}

L'approximation autocohérente permet de donner une solution approchée de l'équation (9) . Plusieurs modèles essayent de relier les paramètres locaux de contrainte et de déformation aux grandeurs globales en faisant intervenir des tenseurs dits de localisation. La modélisation autocohérente propose une forme particulière du tenseur de localisation dans le cas de cristallites ellipsoïdaux. Son utilisation suppose quelques hypothèses, largement moins rédhibitoires que les hypothèses de Reuss ou de Voigt $[14,15]$ :

- L'agrégat polycristallin est composé de DCD séparés par diverses discontinuités (parois de dislocation, joints de grains, ...) dont on négligera l'influence.

- Le comportement élastoplastique est uniforme (ou homogène) à l'intérieur de chaque DCD. Une telle hypothèse ne permet pas, par nature, de décrire correctement les effets de distorsion liés à un champ inhomogène des déformations internes à un DCD.

- Toutes les interactions entre DCD sont négligées. Ainsi on ne tient pas compte des influences des orientations initiales entre DCD voisins, des interactions induites par la déformation (structures de déformation mésoscopiques: bandes de déformation, cellules de déformation, ...). La modélisation autocohérente ne tient donc pas compte des interactions entre le glissement plastique et les cristallites.

Dans le cadre de la modélisation autocohérente, conformément à la précédente hypothèse d'uniformité du comportement plastique à l'intérieur des grains, le polycristal est supposé être constitué de domaines de volume $V^{I}$ (appelés aussi grain ou cristallites) où les déviations $\delta l(r)$ sont uniformes et s'écrivent:

$$
\underline{\underline{\delta l}}(r)=\sum_{I=1}^{N} \Delta l^{I} . \partial^{I}(r) \quad \text { où } \partial^{I}(r)=\left\{\begin{array}{lll}
0 & \text { si } & r \notin V^{I} \\
1 & \text { si } & r \in V^{I}
\end{array}\right.
$$

On suppose de même que le gradient des vitesses locales est défini de la manière suivante:

$$
v_{i, j}^{I}=1 / V^{\prime} \int_{V^{\prime}} v_{i, j}(r) \cdot d V
$$

Ce qui permet d'estimer le champ des vitesses locales par l'approximation :

$$
g_{i j}=v_{i, j}(r)=\sum_{I=1}^{N} v_{i, j}^{I} \cdot \partial^{I}(r)
$$

En reportant ces expressions des grandeurs locales dans l'expression intégrale nous obtenons l'expression suivante du champ de déplacement moyen d'un cristallite quelconque:

$$
g_{i j}^{I}=\underset{\Sigma+\Xi}{g_{i j}^{0}}+\sum_{I=1}^{N} T_{i j k l}^{I J} \cdot \Delta l_{k l m n}^{I} \cdot v_{m, n}^{J} \text { avec } T_{m n o p}^{I J}=1 / V_{i} \iint_{V V_{,}} \Gamma_{m o, p n\left(r-r^{\prime}\right)} d V d V^{\prime}
$$

Le tenseur $\underline{\underline{T}}^{I J}$, appelé tenseur de localisation, décrit les interactions entre les domaines $I$ et $J$. Pour des valeurs de $I=J$, il s'exprime très facilement grâce au tenseur d'Eshelby [16] dans le cas des domaines de forme ellipsoïdale. Cette équation intégrale, qui n'a supposé jusqu'à présent aucune hypothèse, donne une relation "simple" entre le gradient des vitesses de 
déformation local et le gradient des vitesses de déformation du milieu homogène $\underline{\underline{g}}$ qui est pris égal à $G$ (moyenne volumique pondérée des vitesses locales).

\subsection{Description du matériau et du volume diffractant}

Armés des équations de localisation et de la loi de comportement incrémentale des cristallites, nous pouvons calculer, pour tout incrément de chargement les vitesses de déformations locales et les paramètres locaux associés [12]. Il n'est pas possible par contre, d'obtenir en tout point les données locales d'un cristallite, ou d'un quelconque "volume d'arrivée", sans l'avoir suivi dès le début du trajet de chargement et cela incrément par incrément. Usuellement, pour approcher le comportement global du matériaux polycristallin, on crée un ensemble de cristallites appelé pseudo-matériau initialement plus ou moins isotrope dont on suivra l'évolution tout au long du trajet de chargement. Pour conserver l'isotropie initiale du matériau ou du pseudo-matériau, il est nécessaire d'utiliser un nombre très élevé de cristallites pour que quelques uns soient dans un secteur précis de l'espace d'Euler. Typiquement, seulement $1 \%$ des cristaux se trouvent, dans une divergence de $1^{\circ}$, autour de la position de diffraction*. Il est donc nécessaire d'utiliser des pseudos-matériaux d'au moins 2000 à 3000 cristaux pour obtenir un lot de cristallite en position de diffraction statistiquement représentatif (création par générateur pseudo-aléatoire des orientations des cristallites).

Afin de restreindre le nombre total de cristallites et définir de manière précise la distribution des déformations internes des cristallites, nous avons opté pour une solution différente : créer deux ensembles de cristallites : l'un représentatif du matériau global et notamment de sa texture, l'autre représentatif du volume diffractant. Pour chaque incrément de charge, il suffit d'appliquer le calcul au premier pseudo-matériau pour obtenir le comportement "global" et les tenseurs de localisation des vitesses de déformation qui en découlent. Il suffit alors d'appliquer les formules de localisation aux cristallites appartenant au lot représentatif du volume diffractant pour procéder aussitôt au calcul incrémental des paramètres locaux (contraintes, écrouissage, ...). Cette méthode a l'avantage de permettre le calcul précis des données locales de nombreux cristallites situés autour de la position de diffraction pour un nombre de cristallites total minimal. Son seul inconvénient réside dans le fait que les cristallites tournent au cours de la déformation plastique et qu'il faut optimiser le volume échantillonné, pour être représentatif en tout point du trajet de chargement du volume réellement diffractant : le nombre total de cristallite est dès lors légèrement accru.

\section{RESULTATS}

Le rôle des hétérogénéités de déformation d'origine élastique a été étudié en détail par Castex et Sprauel [5, 6, 7]. Ils ont montré que l'anisotropie des caractéristiques élastiques des cristallites entraîne des hétérogénéités assez importantes pour être source d'élargissement. Nous allons vérifier que la déformation plastique entraîne des HDE supérieures à celles rencontrées dans le domaine élastique.

\subsection{Rôle de la déformation plastique et des incompatibilités de déformation}

L'anisotropie due au nombre réduit de systèmes de glissement et les incompatibilités de déformation induites font de la déformation plastique la principale source d'hétérogénéités de déformation élastique. Les déformations plastiques, largement tributaires de l'orientation des cristallites, sont sources d'un champ très hétérogène de contraintes résiduelles triaxial. Afin de vérifier le rôle de la déformation plastique et des incompatibilités qu'elle entraîne, nous avons étudié le comportement d'un matériau au monocristal globalement isotrope : l'aluminium. Les nuages de points de la figure 5 correspondent, pour quatre niveaux de charge, aux positions des

\footnotetext{
${ }^{*} 1,37 \%$ pour un plan $\{\mathrm{hkl}\}$ à multiplicité de 1 .
} 
points déformation plastique locale/contrainte locale (suivant l'axe de traction) des divers cristallites composant le pseudo-matériau. On remarque que les hétérogénéités de déformation élastique (hauteur du nuage) croissent avec la déformation plastique.

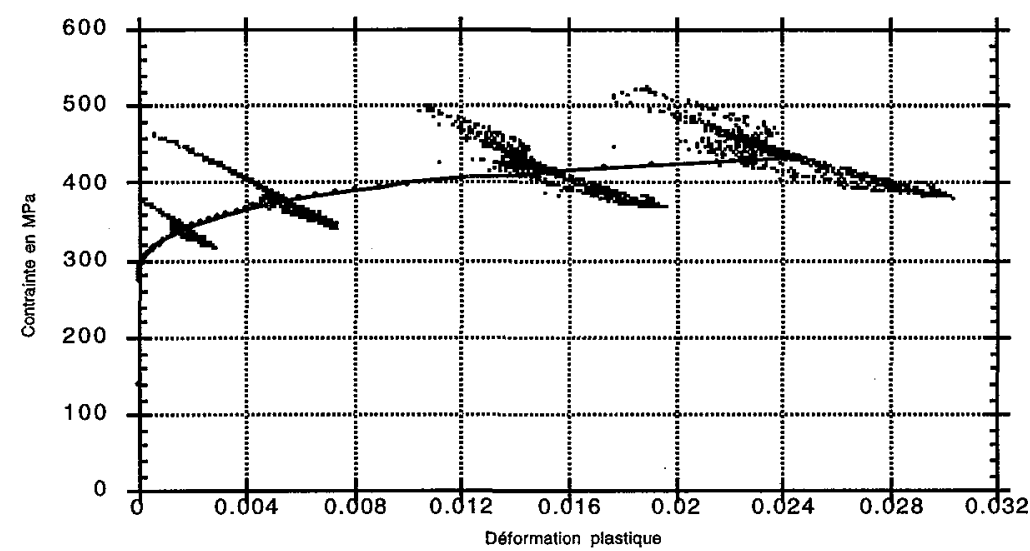

Figure 5 : Simulation de l'essai de traction de l'aluminium. Les nuages de points représentent les contraintes internes obtenues pour quatre niveaux différents de déformation plastique macroscopique, tandis que la courbe continue caractérise l'évolution des contraintes macroscopiques.

Simulated tensile stress-strain curve of polycrystalline aluminum. Scattered points represent the local plastic strain and internal stresses for four different macroscopic load.

\subsection{Evolution des hétérogénéités de déformation du volume diffracant}

Dans le cas d'un matériau au monocristal anisotrope, les fonctions de distribution des hétérogénéités de déformation élastique présentent de profonds changements lors de la plastification. Nous reportons dans la figure 6 l'évolution des déformations moyennes internes aux DCD $\varepsilon_{h k l}(\beta)$, en fonction de la rotation propre $\beta$ autour de la normale au plan diffractant et de la contrainte appliquée. Les caractéristiques élastiques sont celles de l'austénite [5] et le pseudo-matériau est crée de façon à ce que le loi de comportement macroscopique corresponde à celle de l'essais de traction du $316 \mathrm{Ti}$. Avant plastification (vers $280 \mathrm{MPa}$ ), la fonction $\varepsilon_{h k l}(\beta)$ est globalement sinusö̈dale. Après plastification, les évolutions en fonction de $\beta$ sont beaucoup plus "quelconques". Pour le plan (311), $\varepsilon_{h k l}(\beta)$ se caractérise par des amplitudes largement supérieures à celles rencontrées dans domaine élastique (plus de $1.210^{-3}$ à $330 \mathrm{MPa}$ pour 3.7 $10^{-4}$ à $210 \mathrm{Mpa}$ ). Le choix du plan (220) conduit à des amplitudes proches de celles créées dans le domaine élastique. Le plan $\{111\}$ caractérisé par l'homogénéité des déformations $\varepsilon_{h k l}(\beta)$ dans le domaine élastique $[5,6]$, présente des amplitudes du même ordre que celles du $(220)$ dans le domaine plastique : le rôle de ces deux plans dans le glissement plastique entraîne peut être ces faibles évolutions. L'amplitude de la fonction $\varepsilon_{h k l}(\beta)$ pour le plan $\{200\}$ est du même ordre que celle du plan (311), alors que, tout comme le plan $\{111\}$, il est caractérisé par l'homogénéité des $\varepsilon_{h k l}(\beta)$ dans le domaine élastique. Les hétérogénéités rencontrées dans le volume diffractant peuvent, suivant le plan ((311) par exemple) et l'incidence choisis, être aussi importantes que dans l'ensemble du matériau. Le calcul des largeurs de pics de diffraction découle directement du produit de convolution des fonctions de distribution avec les fonctions intensité du pic élémentaire. L'élargissement est d'autant plus grand que l'amplitude des fonctions $\varepsilon_{h k l}(\beta)$ est importante et que l'angle de diffraction $\theta_{h k l}$ est grand. 

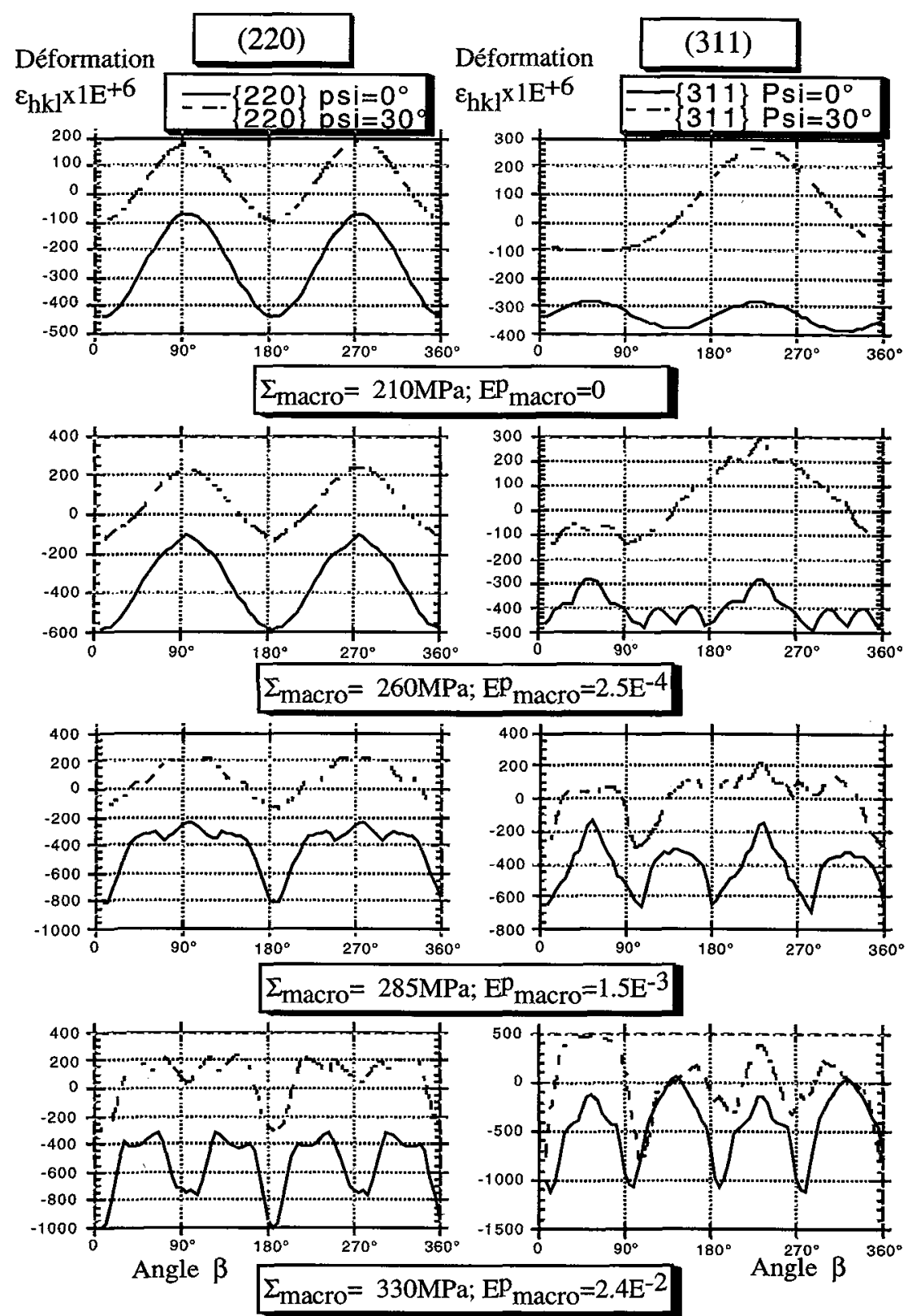

Figure 6 : Evolution de la déformation calculée suivant les normales aux plan (220) et (311) en fonction de la charge appliquée (traction uniaxiale) et de la rotation $\beta$.

Evolution of the function $\varepsilon_{h k l}(\beta)$ of the two planes (220) and (311) versus the applied load. 


\subsection{Résultats expérimentaux}

\subsubsection{Le matériau}

L'alliage 316Ti (ou Z6 CND 17-12) est un acier inoxydable austénitique relativement classique. Sa microstructure est caractérisée par la présence d'alignements de précipités (carbure de titane, ...) dans une matrice à grains relativement grossiers (indice de taille de grain $=7$ ).

\subsubsection{Procédure expérimentale}

Les éprouvettes ont été détensionnées et polies électrochimiquement pour limiter l'influence des contraintes résiduelles d'usinage. Les essais de traction ont été réalisés sur une machine Instron 1185. Les incréments de charge ont été effectués par paliers de 10 à $50 \mathrm{MPa}$. Les acquisitions ont été conduites sous charge avec un diffractomètre SETX suivant les conditions récapitulées dans le tableau 1. La hauteur des pics atteinte dans ces conditions est supérieure à 2500 coups. La largeur intégrale donnée est la moyenne des largeurs obtenues avec chacun des 13 angles d'incidence, l'imprécison statistique est dès lors limitée.

\begin{tabular}{|ll|}
\hline Rayonnement & $\mathrm{Mn} \mathrm{K}_{\alpha}$ \\
Plans diffractants & $(311)$ \\
Directions de mesure & $\phi=0$ (sens long) et $\phi=-45$ \\
Angles Psi & 13 angles $\psi$ entre $+39^{\circ}$ et $-24^{\circ}\left(+/-1^{\circ}\right)$ \\
Surface irradiée & tache de $3 \mathrm{~mm}$ de diamètre \\
Temps d'acquisition & 90 à 120 secondes par pic \\
Détection & Détecteur à localisation linéaire (largeur $\left.16^{\circ}(2 \theta)\right)$ \\
\hline
\end{tabular}

Tableau 1: Conditions de mesure

Experimental setup

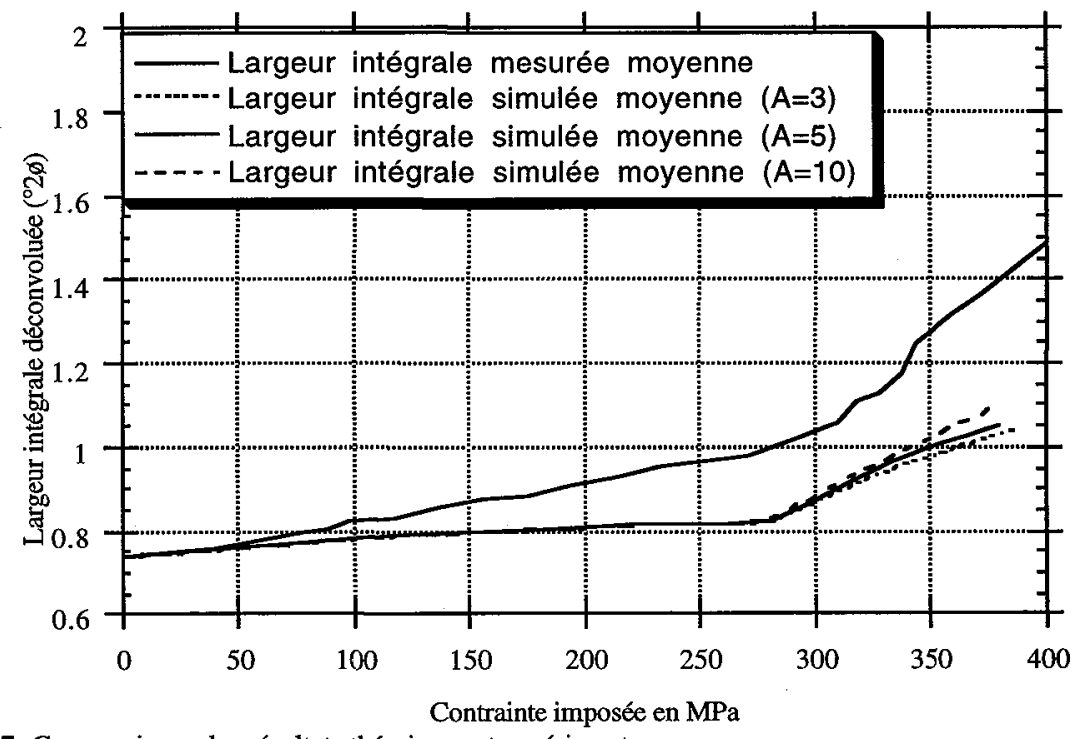

Figure 7: Comparaisons des résuitats théoriques et expérimentaux

Comparison of the experimental and theorical results $(\mathrm{A}=$ Plastic anisotropy parameter $)$ 


\subsubsection{Résultats}

La largeur des pics de diffraction évolue de manière monotone croissante dès le début du domaine d'élasticité (Figure 7). L'évolution dans le diagramme contrainte/largeur intégrale s'effectue suivant deux droites, l'une de pente relativement faible $\left(0,00045^{\circ} 2 \theta / \mathrm{MPa}\right)$ dans le domaine d'élasticité et l'autre de $0,00235^{\circ} 2 \theta / \mathrm{MPa}$ dans le domaine de plasticité $\left(\varepsilon_{\max }=17,4 \%\right)$. La déconvolution de l'élargissement instrumental augmente la sensibilité à l'élargissement et conduit respectivement à des pentes de $0,0008^{\circ} 2 \theta / \mathrm{MPa}$ et $0,0042^{\circ} 2 \theta / \mathrm{MPa}$. La transition entre les droites "élastique" et "plastique" est très nette et se situe aux environs de la limite d'élasticité macroscopique conventionnelle $(\operatorname{Re} \sim 300 \mathrm{MPa})$. L'élargissement observé dans le domaine d'élasticité n'est parfaitement réversible que pour de faibles contraintes $(100 \mathrm{MPa})$ ou après quelques cycles. Cette irréversibilité est sans doute due à la micro-plastification du matériau [6]. A la décharge, le matériau reste sensible à l'évolution des hétérogénéités de déformation et montre une variation significative de la largeur intégrale. La pente rencontrée alors est relativement proche de la pente de charge dans le domaine élastique.

Slope of the different straight lines
Stableau 2: Pentes des differentes droites

Le comportement de la courbe théorique est identique, hormis une légère baisse de la largeur intégrale aux environs de la limite d'élasticité conventionnelle. Cette baisse est due à une diminution des HDE par suite de la plastification des cristallites les plus chargés du volume diffractant. Lors du déchargement élastique, la largeur des pics de diffraction régresse suivant la pente du domaine élastique : les $\mathrm{HDE}$ formées ne diminuent que très partiellement à cause du bloquage dû aux incompatibitlités de déformation plastique. La confrontation des résultats théoriques aux données expérimentales est globalement positive bien que l'amplitude des élargissements calculés (tableau 2) reste en deçà des valeurs expérimentales. Plusieurs raisons peuvent l'expliquer:

- Le choix des lois de comportement élastique et plastique est plus ou moins judicieux. L'anisotropie élastique du monocristal d'austénite varie suivant les alliages et les auteurs $[17,18]$. Les lois d'écrouissage utilisées sont loin d'être rigoureuses et conduisent suivant le choix de certains paramètres à de nettes modifications des HDE. II est possible d'augmenter ou de diminuer à souhait les HDE rencontrées (et donc l'élargissement) en augmentant l'influence de l'écrouissage latent dans la matrice d'écrouissage par l'intermédiaire du coefficient d'anisotropie plastique A. Dans le tableau 2, les résultats théoriques sont donnés pour un coefficient d'anisotropie plastique moyen de 3 [13] en l'absence de données bibliographiques fiables pour l'acier inoxydable 316Ti.

- Le modèle autocohérent n'est qu'une solution approchée de l'équation intégrale (9). Ses hypothèses sont peut être trop restrictives.

- Les effets de taille et de distorsion peuvent se cumuler à l'effet HDE. Cependant, la microstructure des aciers inoxydables austénitiques n'évolue pas de façon importante avant 5\% de déformation. De plus celle-ci est en général due à l'arrangement planaire des dislocations qui à priori donne lieu à peu d'effet de ce type [6].

Les HDE semblent être un facteur d'élargissement important et les comportements mis en évidence ne peuvent donc plus être négligés désormais. Le suivi de la largeur des pics de diffraction $\mathrm{X}$ ne peut plus être considéré comme une méthode d'évaluation du dommage : les élargissements rencontrés sont à priori liés à la conjugaison des effets de taille et distorsion dus à l'évolution de la microstructure et d'un effet $\mathrm{HDE}$ qui n'est lié qu'aux conditions de chargement de la pièce (sollicitation maximale, présence de contraintes résiduelles, ...). 


\section{CONCLUSIONS}

L'étude expérimentale et l'analyse théorique menées sur l'acier inoxydable austénitique 316Ti et développées dans cet exposé tendent à montrer que les hétérogénéités de déformation élastique jouent un rôle important dans l'élargissement des pics de diffraction $\mathrm{X}$ et mettent en évidence l'évolution remarquable de la largeur des pics de diffraction avec la contrainte appliquée. L'élargissement du pic (311) évolue linéairement dans le domaine élastique et dans le domaine plastique. La transition entre domaines plastique et élastique s'effectue par un changement de pente. Les hétérogénéités élastiques de déformation semblent contribuer de façon non négligeable à l'élargissement dans le cas du matériaux et du chargement étudié. L'utilisation des critères classiques de taille et de distorsion semble ici être inappropriée. Cette étude montre qu'en particulier, pour des pièces faiblement sollicitées présentant des contraintes résiduelles macroscopiques, il est peu raisonnable d'interpréter, sans correction, les évolutions des largeurs intégrales des pics de diffraction $\mathrm{X}$ mesurées en surface.

\section{Remerciements}

Les auteurs tiennent à remercier la D.R.E.T pour son soutien actif.

\section{Références}

[1]Warren B.E., Averbach B.L.: J. Appl. Phys., 21(1950) 595.

[2] Warren B.E.,"X ray diffraction " (Addison Wesley, London 1969)

[3]Kröner E.: Modelling Small Deformations of Polycrystals (Elsevier Appl. Sci., London 1986).

[4]Hill R.,"Generalized constitutive relations for incremental deformation of metal crystals by multislip", J. Mech. Phys. Solids, 14 (1966) 95 à 102.

[5]Sprauel J.M., Castex L., "Etude des contraintes d'ordre 2 et 3, Influence sur la forme des pics de diffraction X".Livre de A.Lodini à Paraître.

[6]Sprauel J.M., "Etude par diffraction X des facteurs influençant la corrosion sous contrainte d'aciers inoxydables" (thèse d'état, Paris VI 1988).

[7]Castex L.,"Etude par diffraction X d'aciers au voisinage de la limite d'endurance et à divers stades de la fatigue" (thèse de Doctorat d'Etat, Bordeaux I, 1987).

[8] Taira S., "X-Ray studies on mechanical behavior of materials" (The Society of Materials Science, Japan, 1974)

[9]Reimers W.,"Plastic Deformation of single crystallites in polycrystalline matrices under static loading",

[10]Zaoui A., Séminaire sur les contraintes internes, S.F.M., Voreppe, 1978, 5.

[11]Maeder G., Lebrun J.L., et Sprauel J.M.,"Détermination par diffraction des rayons X des contraintes internes. Aspects macro- et microscopiques" (Ecole d'été d'Oléron, Presses du C.N.R.S.-I.R.S.I.D.)

[12]Iwakuma T. et Nemat-Nasser S.,"Finite elastoplastic deformation of polycrystalline metals", Proc. R. Soc. Lond., A394 (1984) 87-119

[13]Lipinski P., Berveiller M.,"Elastoplasticity of micro-inhomegeneous metals at large strains", Int. J. of Plasticity, 5 (1989) 149-172

[14]Franciosi P. Berveilller M.et Zaoui A., "Latent hardening in copper and aluminum single crystals", Acta Metallurgica, 28 (1980) 273-283.

[14]Voigt W.: Abh. Klg. Ges. Wiss. Göttingen Math. Kl. 34 (1887) 47.

[15]Reuss A.: Z. Ang. Math. Mech., 9 (1929) 49.

[16]Eshelby J.D.: Proc. Roy. Soc., A241 (1957) 376.

[17]François M.,"Détermination des contraintes résiduelles sur des fils d'acier eutectoïde de faible diamètre par DRX" (Thèse ENSAM, 1991 50-51).

[18]Sprauel J.M.,"Détermination des constantes élastiques radiocristallographiques d'un acier inoxydable austénitique" (Thèse de 3 ème cycle, Université de Paris-Sud 1980, 25-35). 